\title{
AREA-AVERAGING OF SURFACE FLUXES IN A NEUTRALLY STRATIFIED, HORIZONTALLY INHOMOGENEOUS ATMOSPHERIC BOUNDARY LAYER
}

\author{
Martin Claussen \\ Forschungszentrum Geesthacht, Postfach 1160, D-2054 Geesthacht, F.R.G.
}

(First received 1 December 1988 and received for publication 4 September 1989)

\begin{abstract}
Different models of effective transfer coefficients of momentum and scalar admixtures for area averaging of surface fluxes over flat, but heterogeneous, terrain are discussed. These proposals are tested by use of a micro-scale numerical model which simulates the response of the surface-layer flow to varying boundary conditions in detail. It is found that sufficiently accurate estimates of effective transfer coefficients can be obtained by averaging the turbulent scales of velocity and concentration based on a 'blending' height. It is suggested that the blending height should be defined as a height at which the sum of deviations of mean flow from local equilibrium and from horizontal homogeneity attains a minimum. This height is of the order of the diffusion height scale which forms a scale for the height up to which the mean flow can be influenced by vertical diffusion.

It is demonstrated that the dispersion of scalar admixtures is particularly sensitive to variations of the surface (or stomatal) resistance, more than to perturbation of the laminar resistance. Furthermore, it is investigated how the subgrid-scale transport terms, which arise due to motions larger than turbulent-scale, but smaller than grid-scale, can affect the calculation of surface fluxes and transfer coefficients.
\end{abstract}

Key word index: Atmospheric surface layer, surface fluxes, area averaging, effective turbulent transfer coefficients, effective drag coefficient, effective roughness, effective laminar resistance, effective surface resistance

\section{INTRODUCTION}

Numerical models of atmospheric flow require parameterization of subgrid-scale transport, i.e. transport by motions which are not resolved by the grid. Current parameterization schemes of subgrid-scale transport within the atmospheric surface-layer usually assume that the surface-layer flow can be treated as a steady state, horizontally homogeneous flow which is in equilibrium with the underlying surface. However, except over vast regions of horizontally homogeneous surface conditions, such as over some portions over the ocean, the atmospheric boundary-layer is rarely an equilibrium flow; instead, it always has to adjust to changing surface conditions. For variations of surface conditions at a sufficiently large scale (say, a few $\mathrm{km}$ ) the surface-layer is commonly supposed to be in a local equilibrium with the lower boundary. This assumption justifies the use of conveniently simple parameterization schemes based on boundary-layer similarity theory. With surface inhomogeneities at small scales, particularly at scales smaller than resolved by the grid, these practical parameterization schemes are no longer applicable. In this case, it is necessary to consider grid averages of surface properties and of flow variables; also, the influence of local advection on the flow structure has to be regarded. Furthermore, it must be taken into account that subgrid-scale transport originates not only from micro-turbulent motions, but also from motions which are induced by heterogeneous terrain.
Recently, various studies have addressed the parameterization of turbulent momentum transfer in heterogeneous terrain with emphasis on the formulation of an 'effective' roughness length. (e.g. André and Blondin, 1986; Wieringa, 1986; Taylor, 1987; Mason, 1988). Although there is no commonly valid definition of an effective roughness length, it is agreed that the effective roughness length is a value intended to represent a spatial average in heterogeneous terrain such that by use of boundary-layer similarity theory a correct spatial average of surface stress or mean velocity is found.

This paper is partly a reassessment of the earlier proposals. Attention is focussed, however, on the formulation of an 'effective' drag coefficient (to be defined in section 4.1.) rather than effective roughness lengths. Some of the earlier concepts are modified. Furthermore, an effective transfer coefficient of scalar admixtures is suggested. Before discussing these proposals, an investigation is made of how subgrid-scale transport terms, which arise due to motions larger than turbulent scale, but smaller than grid-scale, can affect the calculation of surface fluxes and transfer coefficients.

The parameterization schemes proposed in this study are tested by use of a microscale model, which resolves the variation of surface-layer structure due to terrain inhomogeneities in detail, and by interpreting the entire flow domain of the micro-scale model (or a part of it) as one surface-layer grid box of a hypothetical larger-scale model. 
The micro-scale model is briefly presented in section 2. Subsequently, in section 3 , examples of flow modification due to spatially varying surface conditions are given in order to outline important mechanisms of local advection and to point at the differences between local momentum and mass advection. In section 4 , the surface-layer parameterization for transfer coefficients will be discussed.

\section{THE MICRO-SCALE MODEL}

\subsection{Basic equations}

The flow to be studied is a steady state, incompressible, inviscid turbulent flow at neutral stratification. The flow is assumed to be horizontally homogeneous in $y$-direction. The flow region is confined to the atmospheric surface-layer in which the influence of Earth's rotation on the flow is neglected. The basic equations of the mean flow are:

$$
\begin{aligned}
& \frac{\partial U}{\partial x}+\frac{\partial W}{\partial z}=0 \\
& U \frac{\partial U}{\partial x}+W \frac{\partial U}{\partial z}+\frac{\partial \overline{u u}}{\partial x}+\frac{\partial \overline{u w}}{\partial z}=-\frac{1}{\rho_{0}} \frac{\partial P}{\partial x} \\
& U \frac{\partial W}{\partial x}+W \frac{\partial W}{\partial z}+\frac{\partial \overline{u w}}{\partial x}+\frac{\partial \overline{w w}}{\partial z}=-\frac{1}{\rho_{0}} \frac{\partial P}{\partial z} \\
& U \frac{\partial C}{\partial x}+W \frac{\partial C}{\partial z}+\frac{\partial \overline{u c}}{\partial x}+\frac{\partial \overline{w c}}{\partial z}=0 .
\end{aligned}
$$

$x$ and $z$ are horizontal and vertical coordinates, $U$ and $W$ are the mean horizontal and vertical velocities, $C$ is the mean concentration of a passive scalar, $u, w$ and $c$ are the fluctuating components, being defined as differences of actual and mean quantity, $\overline{u w}, \overline{u u}, \overline{w w}, \overline{u c}$, and $\overline{w c}$ are their covariances and autocovariances, $P$ is the mean hydrodynamic pressure, and $\rho_{0}$ is the density of the fluid.

Turbulent momentum fluxes are calculated by using Boussinesq's approach (see Hinze, 1975):

$$
-\overline{u_{i} u_{j}}=K_{m}\left(\frac{\partial U_{i}}{\partial x_{j}}+\frac{\partial U_{j}}{\partial x_{i}}\right)-\frac{2}{3} E \delta_{i j} .
$$

$E$ is the turbulent kinetic energy:

$$
E=\frac{1}{2} \overline{u_{i} u_{i}}
$$

(In Equation 3, Cartesian tensor notation and the Einstein summation convention (see Lumley and Panofsky, 1964) are used. Subscript '1' refers to the $x$ component and subscript ' 2 ' to the $z$-component of a vector.) The turbulent fluxes of passive constituents are parameterized by

$$
-\overline{u_{i} c}=\alpha_{c} K_{m}\left(\frac{\partial C}{\partial x_{i}}\right)
$$

where $\alpha_{c}$ is the inverse turbulent Schmidt number. Since in this study no particular tracer is considered, $\alpha_{c}$ $=1$ is chosen.

For the exchange coefficient $K_{m}$ the so called $E-\varepsilon$ (also known as $k-\varepsilon$ ) model is used, hence

$$
K_{\mathrm{m}}=c_{0}^{4} \frac{E^{2}}{\varepsilon}
$$

where $\varepsilon$ is the dissipation of turbulent kinetic energy. In contrast to other first-order turbulence closures, the $E-\varepsilon$ model realistically simulates a slow relaxation of eddy viscosity to varying surface conditions (Claussen, 1988). Considering the deviation of the surface-layer flow from local equilibrium, the $E-\varepsilon$ model yields results similar to that of a second-order closure model, but it is less complicated to handle.

Equations $1 \mathrm{a}-\mathrm{c}$ are solved by using a stream function-vorticity model. Details of the numerical model including specification of constants are given in Claussen $(1987,1988)$. The boundary conditions to Equations 1a-c are discussed in detail by Claussen $(1987,1988)$. Here, only the boundary conditions for Equation 2 will be given.

\subsection{Boundary conditions}

The lower boundary condition is specified at some height $z=z_{\mathrm{r}}$ at or slightly above the largest roughness length within the flow domain. Varying surface elevation is not taken into account. Within the first grid box above $z=z_{\mathrm{r}}$, which has a vertical depth of a few $\mathrm{cm}$, the flow is assumed to be in equilibrium with its local roughness and surface resistance. Hence,

$$
C\left(z_{\mathrm{r}}\right)=\frac{c_{*}}{\kappa} \ln \left(\frac{z_{\mathrm{r}}}{z_{0 \mathrm{c}}}\right)+c_{*} u_{*} r_{\mathrm{s}}
$$

where $u_{*}$ is the friction velocity, $c_{*}$ is the turbulent concentration scale, and $r_{s}$ is the surface resistance which is a measure for the resistance against transport of an admixture into a surface. $z_{0 c}$ is the so-called roughness length for concentration. The numerical value of $z_{0 c}$ depends on the pollutant as well as the surface characteristics. Over surfaces covered with a vegetation or with similar porous or fibrous roughness elements, $\ln \left(z_{0} / z_{0 c}\right)$ is approximately a constant, whereas over surfaces with bluff roughness elements, $\ln \left(z_{0} / z_{0 \mathrm{c}}\right)$ depends on $u_{*}$ and $z_{0}$. In keeping with Brutsaert (1979) and Hicks (1985)

$$
\ln \left(\frac{z_{0}}{z_{0 c}}\right)=2.3
$$

is chosen for vegetated surfaces and

$$
\ln \left(\frac{z_{0}}{z_{0 c}}\right)=2.9 R e_{*}^{1 / 4} S c^{1 / 2}-2
$$

for surfaces covered with bluff roughness elements. $S c$ is the molecular Schmidt number and $R e_{*}=u_{*} z_{0} / v$ is the friction Reynolds number. 
For convenience, Equation 6 can also be written as

$$
C\left(z_{r}\right)=c_{*} u_{*}\left(r_{\mathrm{a}}\left(z_{\mathrm{r}}\right)+r_{\mathrm{b}}+r_{\mathrm{s}}\right) .
$$

This equation defines the aerodynamic resistance $r_{\mathrm{a}}$ and the laminar resistance $r_{\mathrm{b}}$.

As upstream condition it is assumed that the entire boundary-layer is in equilibrium with the underlying surface. Thus, the upstream conditions are specified at a distance $x_{u}$ sufficiently far upstream of any change in surface roughness or surface resistance where $x_{u} / z_{0} \sim 600$ at least (compare with Claussen, 1987).

The dimensions of the flow domain are chosen such that the upper boundary is at least twice as deep as any internal boundary-layer of velocity or concentration. Thus, it seems warranted to use the unperturbed upstream values as upper boundary values.

The mesh spacing is a few $\mathrm{cm}$ close to the ground and close to roughness transitions. The grid mesh is stretched linearly by $5 \%$ from one grid box to the next as height and distance from a roughness transition increase.

\section{EXAMPLES OF LOCAL MOMENTUM AND MASS ADVECTION}

\subsection{Momentum advection}

A neutrally stratified, steady state flow is considered which passes over a strip of modified surface roughness. The strip with a roughness length $z_{0 F}=0.01 \mathrm{~m}$ is embedded in an elsewhere homogeneous surface with a roughness length $z_{00}=0.001 \mathrm{~m}$. At a height $z=H$ $=10 \mathrm{~m}$, the inflow velocity is $U(H)=1 \mathrm{~m} \mathrm{~s}^{-1}$.

Figure 1 shows the isolines of percentage deviation of local horizontal mean velocity from its unperturbed upstream value. Above the strip of modified roughness, which is located at $-10 \mathrm{~m} \leqslant x \leqslant 0 \mathrm{~m}$, as well as upstream and downstream of it, a deceleration of mean flow is observed. The velocity-defect wake, i.e. the isoline of $1 \%$ deceleration, reaches a maximum height of $h_{U} \simeq 1.3 \mathrm{~m}$ and has a maximum horizontal extent of $x_{\mathrm{U}} \simeq 67 \mathrm{~m}$ downstream of the roughness

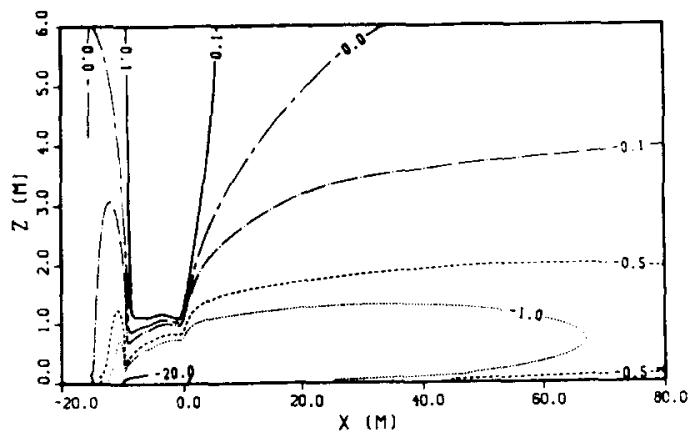

Fig. 1. Isolines of percentage relative difference between perturbed, local and unperturbed, upstream horizontal mean velocity. The flow configuration: the air passes over a strip of modified surface roughness with $z_{\text {OF }}=10^{-2} \mathrm{~m}$ located at $-10 \mathrm{~m} \leqslant x \leqslant 0 \mathrm{~m}$. The strip is embedded in an elsewhere homogeneous surface with $z_{00}=10^{-3} \mathrm{~m}$ modification. A detailed study of $h_{\mathrm{U}}$ and $x_{\mathrm{U}}$ with an assessment to their scaling properties has been reported in an earlier paper (Claussen, 1989). Besides the deceleration of mean flow, an acceleration is noticeable above the strip of modified roughness. The upstream deceleration as well as the acceleration have been discussed earlier (Claussen, 1987). It was shown that upstream deceleration and acceleration is caused by a perturbation of mean pressure. The internal boundary-layer of horizontal mean velocity was found to extend to roughly $x / z_{0 \mathrm{~m}} \sim 300$ upstream of a roughness transition where $z_{0 m}$ is the larger one of upstream and downstream roughness length.

At sufficiently large distances downstream of a roughness transition the modification of mean flow due to a perturbation of mean pressure gradient can be neglected in comparison with advection and diffusion. Considering the turbulent diffusion of momentum, the predicted depth of an internal boundary-layer as well as the structure of vertical profiles of mean velocity and turbulent momentum flux strongly depend on the parameterization of eddy viscosity. Empirical (Beljaars et al., 1983) and theoretical (Claussen, 1988) studies brought forth that eddy viscosity adjusts very slowly to changing surface conditions. By comparison of different models of eddy viscosity it was seen (Claussen, 1988) that the simultaneous response of mixing-length and mixing-velocity (e.g. the square root of turbulent kinetic energy) to a perturbation of surface roughness accounts for the slow relaxation of eddy viscosity. Models of eddy viscosity which do not allow for a variation of mixing length would yield an unrealistically strong growth of an internal boundary and too small a deviation of the flux-profile relationship from equilibrium.

\subsection{Mass advection}

In Fig. 2 isolines of percentage deviation of local mean concentration from upstream equilibrium are plotted. The flow situation is the same as for Fig. 1. As lower boundary condition a constant $z_{0} / z_{0 c}$, i.e. Equation $7 \mathrm{a}$, and a zero surface resistance is chosen.

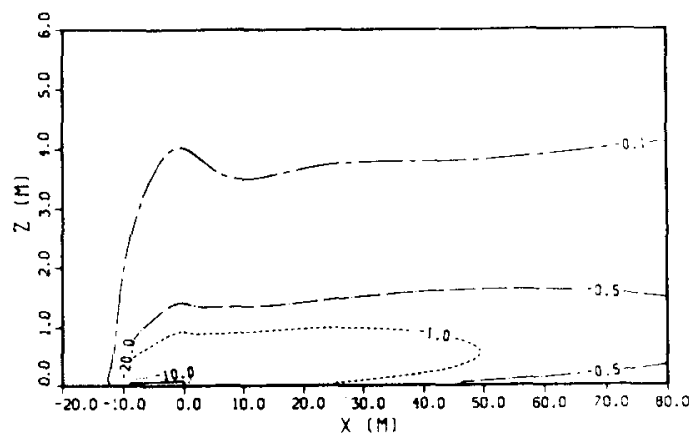

Fig. 2. Isolines of relative difference between perturbed, local and unperturbed, upstream mean concentration of a scalar admixture. The flow configuration is the same as in Fig. 1 and $\ln \left(z_{0} / z_{0 c}\right)=2.3$ and $r_{\mathrm{s}}=0 \mathrm{~s} \mathrm{~m}^{-1}$. 
The shape of the concentration wake is similar to the velocity-defect wake in Fig. 1 except for the flow region close to the roughness transitions. The obvious reason for this difference is that the transport of a passive tracer is not directly affected by the mean pressure, in contrast to momentum. The maximum height and maximum horizontal extent of the concentration wake are approximately $25 \%$ smaller than that of the velocity-defect wake. Of course, height and horizontal extent of the concentration wake depend on the turbulent Schmidt number $1 / \alpha_{c}$. For a Schmidt number larger than unity, the concentration wake would be shorter and shallower than in this simulation. As an example, an increase of the turbulent Schmidt number by a factor of 2 reduces internal boundary-layer height by a factor of approximately $25 \%$.

The qualitative picture provided in Fig. 2 changes drastically, if the surface resistance is altered. In Fig. 3 the isolines of percentage deviation of concentration from upstream equilibrium are depicted for the case of very large surface resistance at the strip of modified roughness. Since this surface virtually blocks vertical flow of contaminant, the concentration is enhanced and the concentration-excess is advected downstream. Moreover, the interaction of blocking of downward transport and advection leads to an accumulation of admixture close to the surface such that, locally, a vertical turbulent flux counter to the upstream flux results.

This example demonstrates that the dispersion of passive admixtures is more sensitive to the surface resistance $r_{\mathrm{s}}$ than to the aerodynamic $r_{\alpha}\left(z_{\mathrm{r}}\right)$ and laminar $r_{\mathrm{b}}$ resistance, simply because the former can be much larger than the latter.

\section{SUBGRID-SCALE PARAMETERIZATION}

\subsection{General remarks}

Because of finite discretization in numerical models the flow is divided into the resolved or grid-scale part and the unresolved or subgrid-scale part. Thus, a mean quantity - theoretically an ensemble average-

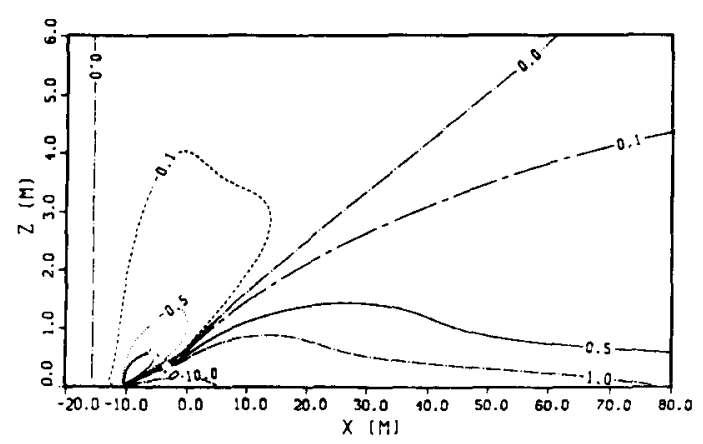

Fig. 3. Same as Fig. 2, except for $r_{\mathrm{s}}=10^{5} \mathrm{~s} \mathrm{~m}^{-1}$ at $-10 \mathrm{~m} \leqslant x \leqslant 0 \mathrm{~m}$. is partitioned as

$$
U(x, y, z)=\{U\}+U^{+}(x, y, z)
$$

where \{\} symbolizes the volume-average over a grid box and the superscript '+' refers to a deviation from grid box average, i.e. to motions at scales smaller than resolved by the grid. Considering the so-called 'control-volume approach' in numerical modeling (See Anderson et al., 1984), conservative quantities are formulated as averages over a grid volume and transporting fluxes (advective and diffusive) over the surfaces enveloping the grid volume. Other finite-difference methods define variables at discrete points; however, the above problem remains, because discrete quantities must be interpreted as somehow averaged values. Similar problems occur in the interpretation of observational data.

In this study, turbulent fluxes from the earth's surface into the lowest atmospheric grid box of a model are considered. The local surface-layer fluxes are often parameterized by a bulk formula (e.g. Louis, 1979):

$-\overline{w c_{0}}(x, y)=C_{\mathrm{s}}(x, y, z) U(x, y, z)\left(C(x, y, z)-C_{0}(x, y)\right)$

where $C_{\mathrm{s}}$ is a bulk transfer coefficient and the subscript ' 0 ' refers to a value at the Earth's surface. In the case of turbulent transports of matter, $C_{\mathrm{s}}$ is often called Dalton number, for momentum transfer, drag coefficient; for the latter, the index ' $s$ ' is replaced by an index 'd'. In a numerical model an 'effective' transfer coefficient $\hat{C}_{\mathrm{s}}$ can be defined by

$$
\left[-\overline{w c_{0}}\right]=\hat{C}_{s}\{U\}\left(\{C\}-\left[C_{0}\right]\right)
$$

where [ ] indicates an area-average over a part of the surface enveloping a grid box, here, over the bottom of the first atmospheric grid box. The value of the effective transfer coefficient depends on the flow configuration and on the numerical grid. In the following section, it will be shown how subgrid-scale motions other than micro-turbulence must be taken into consideration when calculating an average surface flux and how this might affect the formulation of an effective transfer coefficient.

\subsection{Average transfer coefficients}

In his study of turbulent heat flux at the earth's surface, Mahrt (1979) demonstrates that the effective and grid-averaged transfer coefficients of heat transport differ considerably. Here, it will be investigated whether the same is true for the transfer coefficient of a passive tracer and for the drag coefficient.

Averaging Equation 10 by using the partition as in Equation 9, it is found that

$$
\begin{aligned}
{\left[-\overline{w c_{0}}\right]=} & \left\{C_{\mathrm{s}}\right\}\{U\}\{\Delta C\}+\left\{C_{\mathrm{s}}\right\}\left\{U^{+} \Delta C^{+}\right\} \\
& +\{\Delta C\}\left\{C_{\mathrm{s}}^{+} U^{+}\right\}+\{U\}\left\{C_{\mathrm{s}}^{+} \Delta^{\mathrm{c}+}\right\} \\
& +\left\{C_{\mathrm{s}}^{+} U^{+} \Delta C^{+}\right\}
\end{aligned}
$$


with $\Delta C=\left(C-C_{0}\right)$. For turbulent momentum flux it is deduced that

$$
\begin{aligned}
{\left[-\overline{u w_{0}}\right]=} & \left\{C_{\mathrm{d}}\right\}\{U\}^{2}+\left\{C_{\mathrm{d}} U^{+} U^{+}\right\} \\
& +2\{U\}\left\{C_{\mathrm{d}}^{+} U^{+}\right\}+\left\{C_{\mathrm{d}}^{+} U^{+} U^{+}\right\}
\end{aligned}
$$

where $C_{\mathrm{d}}$ is the drag coefficient. In Fig. 4, the correlation terms at the right-hand side of Equation 13 are presented for the same flow configuration as in Fig. 1. It is seen that $\left\{C_{\mathrm{d}}\right\}\{U\}^{2}$ is by far the largest term. The second largest term is $\{U\}\left\{C_{d}^{+} U^{+}\right\}$. Since the latter term is negative, $\left\{C_{\mathrm{d}}\right\}$ overestimates $\hat{C}_{\mathrm{d}}$, in the present case by $10-70 \%$.

Although Fig. 4 refers to just one special flow configuration and although $\left\{C_{\mathrm{d}}\right\}$ as well as $\hat{C}_{\mathrm{d}}$ depend on the specification of depth $\Delta z$ and horizontal extent $\Delta x$ of the grid box, the qualitative picture provided in Fig. 4 is similar for different $\Delta x$ and for other, quite different surface conditions such as single step changes in surface roughness, multiple strips of modified roughness, and randomly varying roughness. $\left\{C_{\mathrm{d}}\right\} \geqslant \hat{C}_{\mathrm{d}}$ is generally observed. As a practical consequence, when formulating a parametric model of $\hat{C}_{\mathrm{d}}$, it would be unwise to seek a parameterization of a local $C_{\mathrm{d}}$ and subsequently to average this local parameterization. Instead, parameterization of $\left[u w_{0}\right]$ and $\{U\}$ should be found from which $\hat{C}_{\mathrm{d}}$, can be calculated.

In Fig. 5 the terms at the right-hand side of Equation 11 are plotted for a flow configuration as in Fig. 2. Here, $\{\Delta C\}\left\{C_{\mathrm{s}}^{+} U^{+}\right\}$and $\{U\}\left\{C_{\mathrm{s}}^{+} \Delta C^{+}\right\}$are the largest subgrid-scale correlations which lead to too large an estimate of $\hat{C}_{\mathrm{s}}$ by $\left\{C_{\mathrm{s}}\right\}$. Again, this result can be generalized. The only exception from the above 'rule' was found for a flow situation in which the surface

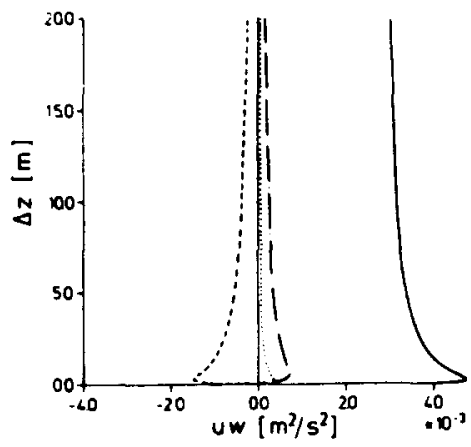

Fig. 4. Various contributions to the grid-averaged surface momentum flux (dimension $\left[\mathrm{m}^{2} \mathrm{~s}^{-2}\right]$ ) as function of the vertical depth of a surface-layer grid box. The flow configuration is the same as in Fig. 1, the horizontal extent over which the surface average is taken, i.e. the horizontal size of the surface-layer grid box is $-15 \mathrm{~m} \leqslant x \leqslant 5 \mathrm{~m}$. The various terms are (see right-hand side of Equation 13): full line: $\left\{C_{d}\right\}\{U\}^{2}$, dashed line: $\{U\}\left\{C_{\mathrm{d}}^{+} U^{+}\right\}$, chaindotted line: $\left\{C_{\mathrm{d}}\right\}\left\{U^{+} U^{+}\right\}$, and dotted line $\left\{C_{d}^{+} U^{+} U^{+}\right\}$.

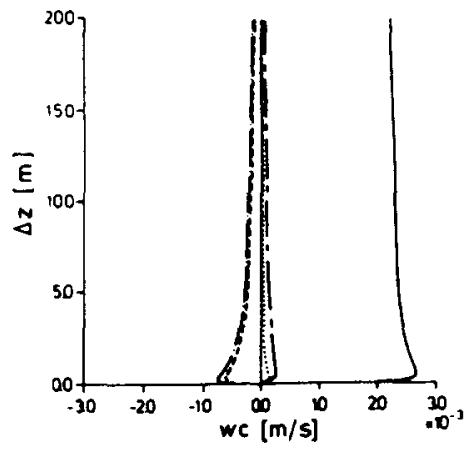

Fig. 5. Same as Fig. 4, except for the surface flux of a scalar admixture. The flow configuration is the same as in Fig. 2. The various terms are (see righthand side of Equation 12): Full line: $\left\{C_{\mathrm{s}}\right\}\{U\}\{\Delta C\}$, chain-dotted line: $\{\Delta C\}$ $\left\{C_{\mathrm{s}}^{+} U^{+}\right\}$, dashed line: $\{U\}\left\{C_{\mathrm{s}}^{+} \Delta C^{+}\right\}$, chain-dashed line: $\left\{C_{\mathrm{s}}\right\}\left\{U^{+} \Delta C^{+}\right\}$, and dotted line: $\left\{C_{s}^{+} U^{+} \Delta C^{+}\right\}$.

resistance varies drastically (i.e. $0 \mathrm{~s} \mathrm{~m}^{-1} \leqslant r_{\mathrm{s}}$ $<10^{5} \mathrm{~s} \mathrm{~m}^{-1}$ ).

By contrast with Mahrt's (1987) results, the subgridscale correlation terms in turbulent transports of momentum and matter in a neutrally stratified atmosphere are much smaller than those in heat transfer; but still they also are not negligibly small.

\subsection{Parametric drag coefficients}

4.3.1. Formulation. In a horizontally homogeneous surface-layer, the local drag coefficient is given by

$$
C_{\mathrm{d}}(z)=\frac{-\overline{u w_{0}}}{U^{2}}=\left(\frac{\kappa}{\ln \frac{z}{z_{0}}}\right)^{2}
$$

and the effective drag coefficient is

$$
\hat{C}_{\mathrm{d}}\left(z_{\mathrm{p}}\right)=\left(\frac{\kappa}{\ln \frac{z_{\mathrm{p}}}{z_{0}}}\right)^{2},
$$

with $z_{\mathrm{p}}$ given by

$$
\ln \frac{z_{\mathrm{p}}}{z_{0}}=\int_{z_{0}}^{\Delta z} \ln \frac{z}{z_{0}} \mathrm{~d} z
$$

where $\Delta z$ is the vertical depth of a surface-layer grid box. $z_{p}$ is the reference height of the effective drag coefficient. From sensitivity studies (not presented here) it turns out that $z_{\mathrm{p}}$ does not strongly depend on $z_{0}$. Thus, in the case of heterogeneous roughness, an approximate guess of some average $z_{0}$ should give a sufficiently accurate value of $z_{p}$, so that the error in $\hat{C}_{\mathrm{d}}$ is negligibly small.

Equation 15 indicates that $\hat{C}_{\mathrm{d}}$ depends on the parameter $z_{0}$ (besides on a reference height $z_{\mathrm{p}}$ ). Mahrt (1987) mentions that one common error in the formulation of subgrid fluxes is the approximation of transfer coefficients in terms of grid ${ }^{2}$ averaged parameter, in 
this case of $\hat{C}_{\mathrm{d}}$ in terms of grid-averaged $z_{0}$, i.e.

$$
\hat{C}_{\mathrm{d}, 1}=\left(\frac{\kappa}{\ln _{\left[z_{0}\right]}^{\frac{z_{p}}{2}}}\right)^{2} \text {. }
$$

Of course, this approach is wrong. It will be interesting to see how wrong it is.

A more sophisticated approach is the use of some effective roughness length. Taylor (1987) proposes that one practical definition of an effective roughness length is given by

$$
\ln z_{0 \mathrm{~m}}=\left[\ln z_{0}\right] .
$$

This $z_{0 \mathrm{~m}}$ is supposed to be an approximation to a more refined effective roughness length $\hat{z}_{0 \mathrm{~m}}$ given by

$$
\ln \hat{z}_{0 \mathrm{~m}}=\frac{\left[u_{*} \ln z_{0}\right]}{\left[u_{*}\right]} .
$$

$\left[u_{*} \ln z_{0}\right]$ and $\left[u_{*}\right]$ are calculated from Rossby number similarity. $\hat{z}_{0 \mathrm{~m}}$ is defined to ensure a correct average velocity profile by assuming that the surfacelayer flow is always in a local equilibrium, hence,

$$
\begin{aligned}
\{U\}= & \frac{1}{\kappa}\left(\left[u_{*}\right] \ln z_{\mathrm{p}}-\left[u_{*} \ln z_{0}\right]\right)=\frac{1}{\kappa}\left(\left[u_{*}\right] \ln z_{\mathrm{p}}\right. \\
& \left.-\left[u_{*}\right] \ln z_{0 \mathrm{~m}}\right) \\
& \simeq \frac{1}{\kappa}\left(\left[u_{*}\right] \ln z_{\mathrm{p}}-\left[u_{*}\right] \ln z_{0 \mathrm{~m}}\right) .
\end{aligned}
$$

Taylor (1987) reports that $\hat{z}_{0 \mathrm{~m}}$ and $z_{0 \mathrm{~m}}$ only slightly differ except for extreme roughness transitions (e.g. $\hat{z}_{0 \mathrm{~m}} / z_{0 \mathrm{~m}} \sim 6$ in the case of a grid square with equal halves with $z_{01}$ and $z_{02}$ and $\left.z_{01} / z_{02}=10^{4}\right)$. Use of $\hat{z}_{0 \mathrm{~m}}$ instead of $z_{0 \mathrm{~m}}$ may pose a serious problem. Since $\hat{z}_{0 \mathrm{~m}}$ depends on the actual flow field, the averages $\left[u_{*} \ln z_{0}\right]$ and $\left[u_{*}\right]$ have to be performed at each time step. This average can be quite expending, if inventories of surface conditions with a high resolution are used. (Such an inventory of the F.R.G. contains several million surface pixels.) The calculation of $\hat{z}_{0 \mathrm{~m}}$ is problematic also from the physical point of view, because the parameters implicit in the Rossby number similarity are hard to determine (see Hasse, 1976). Therefore, with Equation 18 in its approximate form, a conveniently simple parametric model of $\hat{C}_{\mathrm{d}}$ is

$$
\hat{C}_{\mathrm{d}, 2}=\left(\frac{\kappa}{\ln \frac{z_{\mathrm{p}}}{z_{0 \mathrm{~m}}}}\right)^{2} \text {. }
$$

Implicit in Equation 20 is the assumption that the difference between $\left[u_{*}\right]^{2}$ and $\left[u_{*}^{2}\right]$ is small. (In fact, Taylor (1987) shows that $\left[u_{*}\right]^{2} /\left[u_{*}^{2}\right]$ is always close to unity. Even in the case of a grid square with equal halves with $z_{01}$ and $z_{02}$ and $z_{01} / z_{02}=10^{4},\left[u_{*}\right]^{2} /\left[u_{*}^{2}\right]$ $=0.87$.)

As mentioned, $z_{0 \mathrm{~m}}$ is intended to determine the correct average velocity profile rather than the correct average surface stress; furthermore, it is assumed that the mean flow is in equilibrium with the local surface everywhere in the boundary-layer. In order to circumvent these shortcomings Wieringa (1986) and Mason
(1988) suggest evaluation of an effective roughness length $z_{0 e}$ by averaging surface momentum fluxes based on a 'blending' height $l_{\mathrm{b}}$. Mason (1988) considers the blending height a scale-height at which the flow is approximately in equilibrium with the local surface and also independent of position. Hence, $z_{0 \mathrm{e}}$ can be calculated from

$$
\frac{1}{\left(\ln \frac{l \mathrm{~b}}{z_{0 \mathrm{c}}}\right)^{2}}=\left[\frac{1}{\left(\ln \frac{\mathrm{lb}}{z_{0}}\right)^{2}}\right]
$$

$\left(l_{\mathrm{b}}\right.$ can be viewed as a reference height of an areallyaveraged drag coefficient; $l_{\mathrm{b}}$ does not necessarily coincide with $z_{\mathrm{p}}$, the reference height of the effective drag coefficient.) In order to arrive at a parametric $\hat{C}_{\mathrm{d}, 3}$, which is formally the same as $\hat{C}_{\mathrm{d} .2}$ except for using $z_{0 \mathrm{e}}$ instead of $z_{0 \mathrm{~m}}$, it has to be recognized that $U\left(l_{\mathrm{b}}\right) /\{U\} \simeq \ln \left(l_{\mathrm{b}} / z_{0 \mathrm{e}}\right) / \ln \left(z_{\mathrm{p}} / z_{0 \mathrm{e}}\right)$. The latter assumption is less restrictive than the assumption of local equilibrium everywhere in the surface-layer, because the notion that the velocity profile is logarithmic does not necessarily imply that the flow is in equilibrium with the underlying surface. The logarithmic shape of a velocity profile emerges from a quite general assertation on the hydrodynamic stability of a boundarylayer flow (e.g. Malkus, 1979).

Mason (1988) provides a heuristic model which indicates that roughly $l_{\mathrm{b}} / L_{\mathrm{c}} \sim O\left(10^{-2}\right)$ where $L_{\mathrm{c}}$ is the horizontal scale or wave length of roughness variations. In fact, at $z / L_{\mathrm{c}} \sim O\left(10^{-2}\right)$, a perturbed flow has approached a local equilibrium (e.g. Peterson, 1969), but it has to be questioned, whether the flow is independent of horizontal position. Above $z / L_{\mathrm{c}} \sim$ $O\left(10^{-2}\right)$, the horizontal mean velocity varies strongly with height showing an inflection point in the logarithmic vertical profile, or, downstream of a strip of modified roughness, a pronounced 'shoulder' (e.g. Petersen and Taylor, 1973; Claussen, 1989). Thus, at heights of the order $z \sim O\left(10^{-2}\right) L_{\mathrm{c}}$, the flow experiences perturbations due to upstream roughness transitions and begins to adjust to some equilibrium representing an average over horizontal scales larger than $L_{\mathrm{c}}$. It can be expected that horizontal flow homogeneity is unlikely at levels $z \sim O\left(10^{-2}\right) L_{\mathrm{c}}$. Therefore, a different calculation of the blending height will be proposed in this study.

From numerical simulations of flows over randomly varying roughness (to be presented below) it is found that the deviation of horizontal mean velocity from local equilibrium can be quite large, particularly adjacent to a roughness transition. Considering horizontal averages over scales of several $L_{\mathrm{c}}$, however, the deviation amounts to only a few per cent. This averaged deviation increases with height (see Fig. 6). On the other hand, the variation of horizontal mean velocity with fetch decreases with height, because, due to turbulent diffusion, the influence of subsequent roughness transitions on the flow is less and less recognizable as height increases. Thus, there is a certain height $z_{\min }$ (see Table 1) at which the sum of 


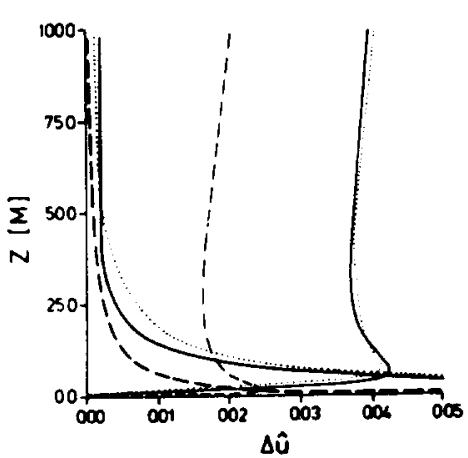

Fig. 6. Averaged deviation of horizontal mean velocity from local equilibrium and horizontal homogeneity.

Thick lines:

$$
\Delta \hat{U}=\frac{[|U(x, z)-[U](z)|]}{[U](z)} .
$$

Thin lines:

$$
\begin{aligned}
& \qquad \Delta \hat{U}=\frac{\left[\left|U(x, z)-U_{0}(x, z)\right|\right]}{U_{0}(x, z)} \\
& \text { with } U_{0}(x, z)=\left(u_{*}(x) / \kappa\right) \ln \left(z / z_{0}(x)\right) \text {. } \\
& \text { The flow configurations which this } \\
& \text { figure refers to is described in section } \\
& \text { 4.3.2.3. Full lines: case WV, dotted lines: } \\
& \text { case UN1, and dashed lines: case UN2. }
\end{aligned}
$$

Table 1.

\begin{tabular}{lccc}
\hline & $z_{\min }(\mathrm{m})$ & $l_{\mathrm{d}}(\mathrm{m})$ & $l_{\mathrm{b}}(\mathrm{m})$ \\
\hline UN2 & 36 & 32 & 3.3 \\
UN1 & 60 & 61 & 7.8 \\
WV & 45 & 62 & 8.1 \\
\hline
\end{tabular}

The 'blending' height calculated from Fig. $6\left(z_{\min }\right)$ and evaluated by Equation $23\left(l_{\mathrm{d}}\right)$ and by Mason's (1988) model $\left(l_{\mathrm{b}}\right)$, Equation 10 in his paper.

both deviations (from local equilibrium and from horizontal homogeneity) attains a minimum. It turns out that this height is roughly as large as the diffusion height scale $z_{\mathrm{d}} \sim L_{\mathrm{c}} u_{*} U\left(z_{\mathrm{d}}\right)$ which forms an asymptotic scale for the height up to which the mean flow can be influenced by vertical diffusion (see Tennekes and Lumley, 1972). No proof is provided that a height $z_{\min }$ always exists. But in the cases for which no $z_{\min }$ can be found, the blending height could be defined as a height at which the sum of the deviations from local equilibrium and horizontal homogeneity becomes smaller than a certain limit value. $z_{\mathrm{d}}$ can be evaluated from

$$
z_{\mathrm{d}} \sim L_{\mathrm{c}} \frac{u_{*}}{U\left(z_{\mathrm{d}}\right)}=L_{\mathrm{c}} \frac{\kappa}{\ln \left(\frac{z_{\mathrm{d}}}{z_{0}}\right)} .
$$

The constant of proportionality is chosen $2 \kappa$ in analogy to Jackson and Hunt's (1975) definition of an inner scale height of wind flow over low hills. A convenient approximation $l_{\mathrm{d}}$ to $z_{\mathrm{d}}$ is given by

$$
l_{\mathrm{d}}=0.7 z_{0}\left(\frac{L_{\mathrm{c}}}{z_{0}}\right)^{4 / 5},
$$

which approximately fits Equation 22 within $10^{2}<L_{\mathrm{c}} / z_{0}<10^{5}$. It turns out that the calculation of an effective roughness length from Equation 21 based on either $l_{\mathrm{b}}$ or $l_{\mathrm{d}}$ is not too sensitive to the exact value of $l_{\mathrm{b}}$ or $l_{\mathrm{d}}$, therefore, the choice of a typical $L_{\mathrm{c}}$ and $z_{\mathrm{o}}$ is not critical.

In Table $1, z_{\min }, l_{\mathrm{d}}$ and $l_{\mathrm{b}}$ are given for the cases shown in Fig. 6. It is obvious that $l_{\mathrm{d}}$ is a better approximation to $z_{\min }$ than $l_{\mathrm{b}} \cdot l_{\mathrm{b}}$ is almost an order of magnitude smaller than $z_{\min }$.

For completeness, a fourth parametric model $\hat{C}_{\mathrm{d} .4}$ is studied which was originally proposed by André and Blondin (1986). André and Blondin (1986) derive an effective roughness length by assuming that the horizontal mean velocity is uniform over horizontal scales of several $L_{\mathrm{c}}$ at the top of the first atmospheric grid box-regardless of the flow configuration. This in general unrealistic assumption brings about that their effective roughness length strongly depends on an arbitrary specification of the vertical grid size, although it should characterize a surface condition rather than a grid mesh.

André and Blondin's (1986) model can be reformulated using the above arguments. By recognizing that at heights of the order $l_{\mathrm{d}}$ the deviation of mean velocity from both local equilibrium and horizontal homogeneity attains a minimum, a sufficiently accurate estimate of $\left[u_{*}\right]$ is given by averaging the inverse of $\ln \left(z / z_{0}\right)$ at $z=l_{\mathrm{d}}$. Then, André and Blondin's (1986) effective roughness length $z_{0 \mathrm{a}}$ revised is

$$
\frac{1}{\ln \frac{\mathrm{ld}}{z_{0 \mathrm{~m}}}}=\left[\frac{1}{\ln \frac{\mathrm{ld}}{z_{0}}}\right] \text {. }
$$

This $z_{0 \mathrm{a}}$ depends only on surface conditions and on the horizontal grid spacing (which effectively mean alteration of surface conditions), but not on the vertical grid size. From Equation 24 and with the assumption that $\left[u_{*}\right]^{2} \simeq\left[u_{*}^{2}\right], \hat{C}_{\mathrm{d}, 4}$ is the same as $\hat{C}_{\mathrm{d}, 2}$ except for using $z_{0 \mathrm{a}}$ instead of $z_{0 \mathrm{~m}}$.

4.3.2. Results. The relative difference of $\hat{C}_{\mathrm{d}, i}(i$ $=1,2,3,4)$ and $\hat{C}_{\mathrm{d}}$ is shown below for different flow configurations.

\subsubsection{Single roughness transition}

A flow is considered which flows from a smooth surface $\left(z_{01}=10^{-5} \mathrm{~m}\right)$ onto a slightly rougher surface $\left(z_{02}=10^{-3} \mathrm{~m}\right)$. The flow domain extends from $x=$ $-10 \mathrm{~m}$ to $x=+10 \mathrm{~m}$ with the roughness transition at $x=0 \mathrm{~m}$. These scales, which are much smaller than a typical meso-scale size of $\Delta x \sim O(\mathrm{~km})$, are chosen in order to investigate the influence of strong local momentum advection on the performance of the parametric $\hat{C}_{\mathrm{d}, i}$. For this flow configuration, $L_{\mathrm{c}}=\Delta x$ $=20 \mathrm{~m}$ is prescribed.

From Fig. 7 it is seen that $\hat{C}_{\mathrm{d}, 1}$ drastically overestimates the effective drag coefficient and, hence, the 


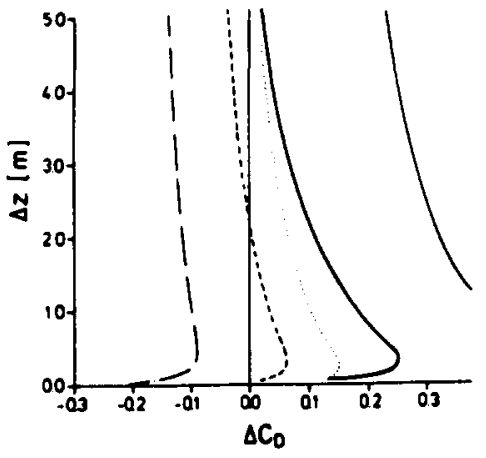

Fig. 7. Relative difference of parametric and effective drag coefficient

$$
\Delta C_{\mathrm{D}}=\frac{\hat{C}_{\mathrm{d}, i}-\hat{C}_{\mathrm{d}}}{\hat{C}_{\mathrm{d}}}
$$

as function of the vertical depth of a surface-layer grid box. Full line: $i=1$, chain-dotted line: $i=2$, dotted line: $i=3$, dashed line: $i=4$. Fat, full line: $i=1,2,3,4$. The flow configuration corresponds to a single step change in surface roughness. For details see section 4.3.2.1.

turbulent momentum flux. $\hat{C}_{\mathrm{d}, 2}$ gives too low an estimate, $\hat{C}_{\mathrm{d}, 3}$ and $\hat{C}_{\mathrm{d}, 4}$ are in between; $\hat{C}_{\mathrm{d}, 4}$ performing better for shallower grid boxes, $\hat{C}_{\mathrm{d}, 3}$, for deeper ones. Quite the same result is found, if $z_{01}$ $=10^{-3} \mathrm{~m}$ and $z_{02}=10^{-5} \mathrm{~m}$ (not shown here). As it will be seen below, the relation

$$
\hat{C}_{\mathrm{d}, 1}>\hat{C}_{\mathrm{d}, 3}>\hat{C}_{\mathrm{d}, 4}>\hat{C}_{\mathrm{d}, 2}
$$

holds generally.

The thick line in Fig. 7 depicts the error of parametric $\hat{C}_{\mathrm{d}, i}$, if the horizontal average is taken over $0<x<10 \mathrm{~m}$. In the latter case, $\hat{C}_{\mathrm{d}, 1}=\hat{C}_{\mathrm{d} .3}=\hat{C}_{\mathrm{d} .4}$ $=\hat{C}_{\mathrm{d}, 2}$, because the roughness length is uniform over the grid mesh. The latter example indicates that the parametric $\hat{C}_{\mathrm{d}, i}$ may give erroneous momentum flu$x e s$, if in a large-scale model no subgrid-scale, but just grid scale, i.e. resolved roughness transitions occur. Furthermore, in the latter case, better estimates of the effective drag coefficients may result (at least when using $\hat{C}_{\mathrm{d}, 3}$ or $\hat{C}_{\mathrm{d}, 4}$ ), if the grid mesh is chosen such that the roughness transitions are located within a grid box, not at their edges. The variation of the errors with the vertical depth $\Delta z$ of a grid box indicates that the assumption $U\left(l_{\mathrm{d}}\right) /\{U\} \simeq \ln \left(l_{\mathrm{d}} / z_{0 \mathrm{e}}\right) / \ln \left(z_{\mathrm{p}} / z_{0 \mathrm{e}}\right)$ is not strictly met. The deviation of mean velocity from a logarithmic profile is largest close to a roughness transition (e.g. Claussen, 1988). As seen below, if larger horizontal grid sizes are considered, then the variation of errors with $\Delta z$ becomes smaller.

\subsubsection{Alternating roughness lengths}

The flow upstream of any perturbations is in equilibrium with $z_{00}=10^{-3} \mathrm{~m}$. Subsequently, the roughness length alternates between $z_{01}=10^{-2} \mathrm{~m}$ and $z_{02}$ $=10^{-4} \mathrm{~m}$ every $100 \mathrm{~m}$, case 1 (thick lines in Fig. 8),

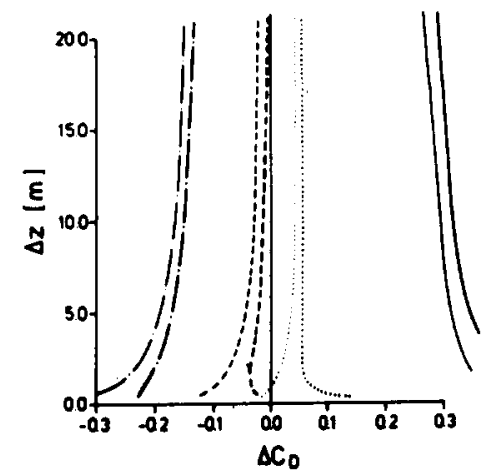

Fig. 8. Same as Fig. 7, except for alternating roughness lengths. The size of patches is the same for different roughness length. For details see section 4.3.2.2.

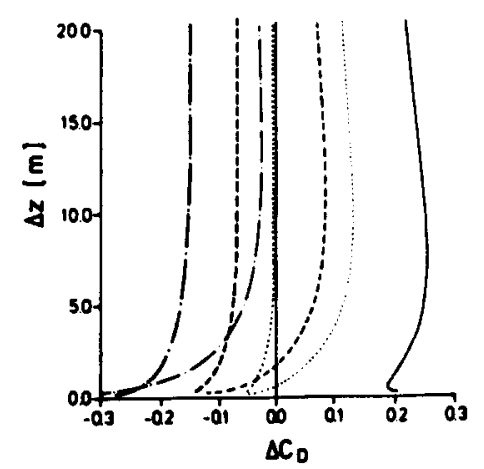

Fig. 9. Same as Fig. 8, except that the size of patches differs for different roughness length. For details see section 4.3.2.2.

and every $10 \mathrm{~m}$, case 2 (thin lines in Fig. 8). Hence, $L_{\mathrm{c}}$ $=200 \mathrm{~m}$ and $L_{\mathrm{c}}=20 \mathrm{~m}$, respectively. A flow configuration like this could be found in the marginal ice zone where ice banding is frequently observed. $z_{00}$ is the roughness length for off-sea wind in coastal areas, while $z_{01}$ is the approximate roughness length of brash (Anderson, 1987) and $z_{02}$, of calm sea. The flow domain over which the horizontal average is performed, i.e. the horizontal grid size of a hypothetical larger-scale model, is $5 \mathrm{~km}$ for case 1 and $500 \mathrm{~m}$ for case 2 . For both cases, $\hat{C}_{\mathrm{d}, 3}$ and $\hat{C}_{\mathrm{d}, 4}$ turn out to be superior to $\hat{C}_{\mathrm{d}, 2}$ and $\hat{C}_{\mathrm{d}, 1}$.

A more difficult flow configuration concerning drag parameterization is the following. From $z_{00}=10^{-4} \mathrm{~m}$ the roughness length increases to $z_{01}=10^{-2} \mathrm{~m}$ for $5 \mathrm{~m}$ (case 1, thick lines in Fig. 9) and for $20 \mathrm{~m}$ (case 2, thin lines in Fig. 9) and subsequently returns to $z_{00}$ for the next $20 \mathrm{~m}$ in case 1 and $5 \mathrm{~m}$ in case 2 . Thus, the flow experiences an increase of surface roughness on the average over the flow domain due to small-scale roughness length variations. The flow domain is $500 \mathrm{~m}$.

For both cases, the error of $\hat{C}_{\mathrm{d}, 4}$ stays within $\pm 10 \%$, except for very small $\Delta z$. The error in $\hat{C}_{\mathrm{d}, 3}$ 
slightly exceeds $+10 \%$. $\hat{C}_{\mathrm{d}, 2}$ generally gives too low an estimate and $\hat{C}_{\mathrm{d} .1}$ is the worst model, its error exceeds $30 \%$ in the case 2 (therefore, not shown in Fig. 9).

\subsubsection{Randomly varying roughness}

Within a flow domain of $5 \mathrm{~km}$, which is again also the horizontal grid size of a larger-scale model, the roughness lengths varies randomly every $100 \mathrm{~m}$. The random roughness length are taken from a positively skewed Weibull distribution (labeled WV in Fig. 10) with shape parameter $a=1.5$ and scale parameter $b$ $=0.06$ and from two uniform distributions (UN1 and $\mathrm{UN} 2$, respectively) with an average roughness length $\left[z_{0}\right]=0.15 \mathrm{~m}$ and $\left[z_{0}\right]=0.005 \mathrm{~m}$, respectively. The average roughness length of the Weibull distribution is $\left[z_{0}\right]=0.15 \mathrm{~m}$. The upstream roughness length is specified $z_{00}=z_{0 \mathrm{~m}}$ (see Equation 18a). As a first guess, $L_{\mathrm{c}}=400 \mathrm{~m}$ is chosen. A choice $L_{\mathrm{c}}=200 \mathrm{~m}$ or $L_{\mathrm{c}}$ $=500 \mathrm{~m}$ alters the results only marginally.

Figure 11 clearly demonstrates that the skill of $\hat{C}_{\mathrm{d}, i}$ in predicting the effective drag coefficient over randomly varying surface roughness is almost independent of the shape of the probability distribution of $z_{0}$. The errors of $\hat{C}_{\mathrm{d}, i}$ in the case UN2 are not shown in Fig. 11, because they are similar to those for UN1, except that the errors in the former are smaller than in the latter case. Again, it can be seen that $\hat{C}_{\mathrm{d} .3}$ and $\hat{C}_{\mathrm{d} .4}$ are superior to $\hat{C}_{\mathrm{d}, 1}$ and $\hat{C}_{\mathrm{d} .2}$. On the other hand, the errors of the latter models are still quite small, if one keeps in mind that an uncertainty in $z_{0}$ of a factor of 2 (or $1 / 2$ ) implies a corresponding error of the drag coefficients of $\pm 30 \%$ for $z_{0} \sim O(0.1 \mathrm{~m})$ (compare with Garratt, 1977).

\subsection{Parametric transfer coefficients of scalar admix-} tures

4.4.1. Constant $z_{0} / z_{0 c}$. Four parametric transfer coefficients $\hat{C}_{\mathrm{s}, i}(i=1,2,3,4)$ are proposed in analogy to the four parametric drag coefficients. For $\hat{C}_{\mathrm{s}, 1}$, which is analogous to $\hat{C}_{\mathrm{d}, 1}$, grid averaged $z_{0}$ and $z_{0 \mathrm{c}}$ are considered, thus,

$$
\hat{C}_{\mathrm{s}, 1}=\frac{\kappa^{2}}{\ln \frac{z_{\mathrm{p}}}{\left[z_{0}\right]} \ln \frac{z_{\mathrm{p}}}{\left[z_{0 \mathrm{c}}\right]}} .
$$

For $\hat{C}_{\mathrm{s}, 2}$, an effective $z_{0 \mathrm{~cm}}$ is defined such that

$$
\hat{C}_{\mathrm{s}, 2}=\frac{\kappa^{2}}{\ln \frac{z_{\mathrm{p}}}{z_{0 \mathrm{~m}}} \ln \frac{z_{\mathrm{p}}}{z_{0 \mathrm{~cm}}}}
$$

with

$$
\ln z_{0 \mathrm{~cm}}=\left[\ln z_{0 \mathrm{c}}\right]
$$

Similarly,

$$
\hat{C}_{\mathrm{s}, 3}=\frac{\kappa^{2}}{\ln \frac{z_{p}}{z_{0 e}} \ln \frac{z_{p}}{z_{0 c e}}}
$$

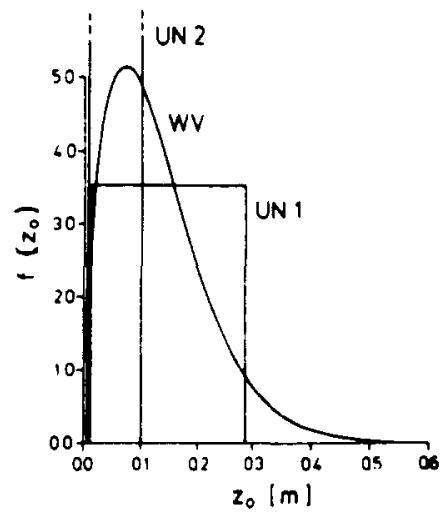

Fig. 10. Probability distribution of $z_{0}$. WV is a two-parameter Weibull distribution with shape parameter $a$ $=1.5$ and scale parameter $b=0.06$; the mean value of $z_{0}$ is $\left[z_{0}\right]=0.15 \mathrm{~m}$. UN1 and UN2 are uniform distributions with $\left[z_{0}\right]=0.15 \mathrm{~m}$ and $\left[z_{0}\right]=0.005 \mathrm{~m}$, respectively.

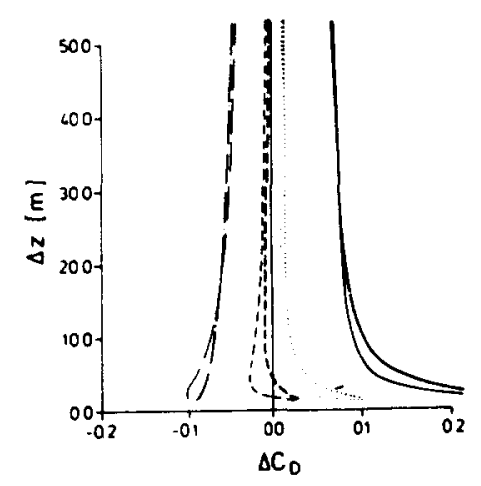

Fig. 11. Same as Fig. 7, except for randomly varying roughness lengths. Thick lines: case UN1, thin lines case WV. For details see section 4.3.2.3.

with

and

$$
\frac{1}{\ln \frac{l d}{z_{0 e}} \ln \frac{l d}{z_{0 c e}}}=\left[\frac{1}{\ln \frac{l d}{z_{0}} \ln \frac{l d}{z_{0 c}}}\right],
$$

$$
\hat{C}_{\mathrm{s}, 4}=\frac{\kappa^{2}}{\ln \frac{z_{\mathbf{p}}}{\left[z_{0 \mathbf{a}}\right]} \ln \frac{z_{\mathbf{p}}}{z_{0 \mathrm{ca}}}}
$$

with

$$
\frac{1}{\ln \frac{l_{\mathrm{d}}}{z_{0 \mathrm{ca}}}}=\left[\frac{1}{\ln \frac{l \mathrm{~d}}{z_{0 \mathrm{c}}}}\right]
$$

is obtained.

The concept of a blending height of mean velocity is also applicable to mean concentration of a scalar admixture. In general, the blending height of mean concentration is of the same order of magnitude as 
that of mean velocity. Therefore, the use of $l_{\mathrm{d}}$ in Equations 27 and 28 is justified. Only in flows over surfaces with drastically varying surface roughness, the blending height of mean concentration exceeds that of mean velocity. However, in this case, more important problems arise than finding the correct blending height.

Provided, that $z_{\mathrm{o}} / z_{\mathrm{0c}}$ is a constant as over vegetated surfaces (Equation 7a), parametric transfer coefficients of scalar admixtures behave like parametric drag coefficients with the exception that the errors in $\hat{C}_{\mathrm{s}, i}(i$ $=1,2,3,4)$ are smaller than $C_{\mathrm{d}, i}(i=1,2,3,4)$. As an example, the results from a flow over randomly varying $z_{0}$ are presented in Fig. 12. The flow configuration is the same as in Fig. 11 using the Weibull $z_{0}$ distribution (case WV).

In the following sections 4.4 .2 and 4.4 .3 only $\hat{C}_{\mathrm{s}, 4}$ is considered, becaúse it seems to be superior to $\hat{C}_{\mathrm{s}, 1}$ and $\hat{C}_{\mathrm{s}, 2} . \hat{C}_{\mathrm{d}, 3}$ could be taken as well; however, calculation of Equation 28 is a little more convenient than Equation 27.

4.4.2. Varying $z_{0} / z_{0 \mathrm{cc}}$. If $z_{0} / z_{\mathrm{oc}}$ remains a constant, there are obviously little problems in providing a parametric transfer coefficient. The problems arise, if $z_{0} / z_{0 c}$ varies with the friction Reynolds number as over surfaces with bluff roughness elements (Equation $7 b)$. In this case averaging over $z_{0 c}$ or functions of $z_{0 c}$ would require a local or subgrid-scale $R e_{*}$, which is unknown to a model.

A practical guess of $\hat{C}_{\mathrm{s}, 4}$ is found by writing

$$
\hat{C}_{\mathrm{s}, 4}=\frac{\kappa^{2}}{\ln \frac{z_{\mathrm{p}}}{z_{0 \mathrm{a}}} \ln \frac{z_{\mathrm{p}}}{z_{0 \mathrm{ca}}}}=\frac{\kappa^{2}}{\ln \frac{z_{\mathrm{p}}}{z_{0 \mathrm{a}}}\left(\ln \frac{z_{\mathrm{p}}}{z_{0 \mathrm{a}}}+\ln \frac{z_{0 \mathrm{a}}}{z_{0 \mathrm{ca}}}\right)}
$$

and by approximating $\ln \left(z_{0 \mathrm{a}} / z_{0 \mathrm{ca}}\right)$ by

$$
\ln \frac{z_{0 \mathrm{a}}}{z_{\text {Oca }}}=2.92\left(\frac{\hat{u}_{*} z_{0 \mathrm{a}}}{v}\right)^{1 / 4}\left[S c^{1 / 2}\right]-2
$$

with

$$
\hat{u}_{*}=\frac{\kappa}{\ln \frac{z_{\mathrm{p}}}{z_{0 \mathrm{a}}}}\{U\}
$$

Equation 30 is, of course, not deduced, it is merely an 'educated' guess which has to be validated by numerical simulations.

Figure 13 depicts the errors in $\hat{C}_{\mathrm{d}, 4}$. The flow configuration are the same as in Fig. 11 using the Weibull $z_{0}$ distribution WV (full line), the uniform $z_{0}$ distribution UN1 (dotted line), and UN2 (dashed line). Cases WV, UN1, and UN2 essentially differ by their friction Reynolds number; for WV and UN1, $30<R e_{*}<1000$ and for UN2, $0.2<R e_{*}<40$. Surprisingly enough, the 'educated' guess is quite good an approximation. From other flow simulations not presented here, it turns out that if $z_{0} / z_{0 c}$ is a constant throughout the flow domain, say $\ln \left(z_{0} / z_{0 c}\right)=\gamma$, then $\ln \left(z_{0 \mathrm{ca}} / z_{0 \mathrm{ca}}\right)=\gamma$ is also a good approximation to Equation $28 \mathrm{~b}$.

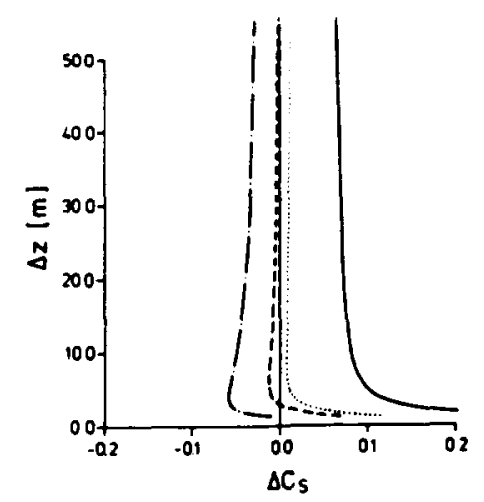

Fig. 12. Same as Fig. 7, except for relative differences of parametric and effective transfer coefficients of scalar admixtures. The flow configuration is the same as for Fig. 11, case WV, and the boundary conditions are $\ln \left(z_{0} / z_{0 c}\right)$ $=2.3$ and $r_{\mathrm{s}}=0 \mathrm{~s} \mathrm{~m}^{-1}$.

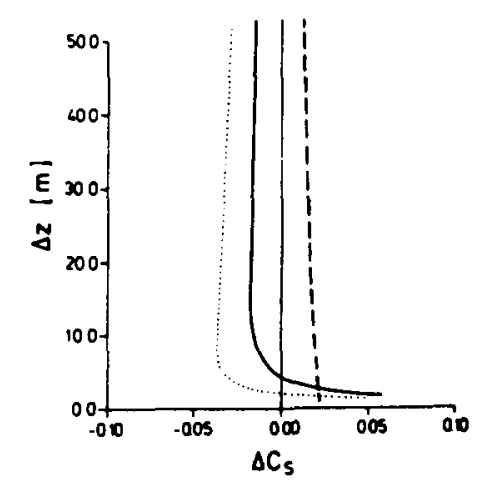

Fig. 13. Relative difference of parametric and effective transfer coefficients of scalar admixtures $\hat{C}_{\mathbf{s}, 4}$ and $\hat{C}_{s}$, as function of the vertical depth of a surface-layer grid box. The flow configurations are the same as for Fig. 11, except for full line: case WV, dotted line: case UN1, dashed line: case UN2, and the boundary condition $r_{\mathrm{s}}$ $=0 \mathrm{~s} \mathrm{~m}^{-1}$ and

$$
\ln \left(\frac{z_{0}}{z_{0 c}}\right)=2.9 R e_{*}^{1 / 4} S c^{1 / 2}-2
$$

4.4.3. Non-zero surface resistance. In the case of non-vanishing surface resistance, the same problem occurs as in the case of varying $z_{0} / z_{0 c}$. In order to find a correct $\left[c_{*}\right]$, the local friction velocity should be known (see Equation 6). If an effective surface resistance $r_{\text {sa }}$ is defined by averaging $c_{*}$ based on a blending height, then, in analogy to Equation $28 \mathrm{~b}$ and with Equation $6, r_{\mathrm{sa}}$ could be calculated from

$$
\frac{1}{\ln \frac{l_{\mathrm{d}}}{z_{\mathrm{O}_{\mathrm{ca}}}}+\kappa u_{*, \mathrm{~g}} r_{\mathrm{sa}}}=\left[\frac{1}{\ln \frac{l_{\mathrm{d}}}{z_{0_{\mathrm{cas}}}}+\kappa u_{*, \mathrm{~g}} r_{\mathrm{s}}}\right] \text {. }
$$


where in ignorance of a local $u_{*}(x)$, some appropriate first guess $u_{*, \mathrm{~g}}$ is taken. Unfortunately, $r_{\mathrm{sa}}$ is quite sensitive to $u_{*, g}$ which makes the use of Equation 32 impractical.

A pragmatic alternative to Equation 32 is given by

$$
r_{\mathrm{sa}}=\left[\frac{1}{r_{\mathrm{s}}}\right]^{-1}
$$

and taking $u_{*, \mathrm{~g}}=\hat{u}_{*}$ (see Equation 31 ). The formulation of an effective surface resistance by Equation 33 basically takes into account that the surface flux of a scalar admixture is inversely proportional to the surface resistance (compare with Equations 6 and 8). The errors of $\hat{C}_{\mathrm{s}, 4}$ with use of Equation 33 are depicted in Fig. 14.

The flow configuration which Fig. 14 refers to is the same as in Fig. 12, except for different probability distributions of $z_{0}$ and $r_{\mathrm{s}}$ listed below.

Case 1, full line: uniform $z_{0}$ distribution UN2 (see Fig. 10), Weibull distribution for $r_{s}$ with shape parameter $a=1$ and scale parameter $b=50$, thus, $\left[r_{\mathrm{s}}\right] \simeq 50 \mathrm{~s} \mathrm{~m}^{-1}$. The upstream surface resistance is $r_{\mathrm{s}}$ $=0 \mathrm{~s} \mathrm{~m}^{-1}$.

Case 2, dotted line: same as Case 1 except for upstream resistance $r_{\mathrm{s}}=\left[r_{\mathrm{s}}\right]=50 \mathrm{~s} \mathrm{~m}^{-1}$.

Case 3, dashed line: same as Case 1, except for the Weibull $z_{0}$ distribution WV (see Fig. 10).

Case 4, chain-dashed line: same as Case 1, except for varying $r_{\mathrm{s}}$ between $r_{\mathrm{s}}=0 \mathrm{~s} \mathrm{~m}^{-1}$, if $z_{0} \leqslant\left[z_{0}\right]$, and $r_{\mathrm{s}}$ $=10^{5} \mathrm{~s} \mathrm{~m}^{-1}$, if $z_{0}>\left[z_{0}\right]$. This specification brings about that $2 / 3$ of the surface is covered with $r_{\mathrm{s}}$ $=0 \mathrm{~s} \mathrm{~m}^{-1}$, and $1 / 3$ with $r_{\mathrm{s}}=10^{5} \mathrm{~s} \mathrm{~m}^{-1}$.

Case 5, chain-dotted line: same as Case 1 except for a Weibull $r_{\mathrm{s}}$ distribution with scale parameter $b=500$, thus, $\left[r_{\mathrm{s}}\right] \simeq 500 \mathrm{~s} \mathrm{~m}^{-1}$.

Two cases in which a very large surface resistance $r_{\mathrm{s}}$ $=10^{5} \mathrm{~s} \mathrm{~m}^{-1}$ was specified for more than half of the flow domain are not shown. In these cases the error of $\hat{C}_{\text {s. } 4}$ varies between $+30 \%$ and $+80 \%$. Obviously, Equation 33 provides a reasonably good estimate of

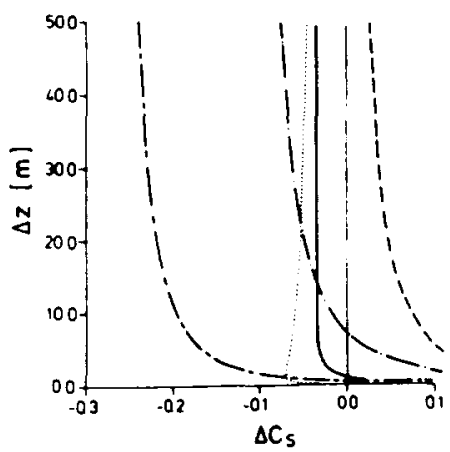

Fig. 14. Same as Fig. 13, except for different flow configurations and boundary conditions $r_{\mathrm{s}} \neq 0 \mathrm{~s} \mathrm{~m}^{-1}$ and $\ln \left(z_{0} / z_{0 \mathrm{c}}\right)=2.3$. The flow configurations are described in detail in section 4.4.3. an effective surface resistance for flow configurations in which the surface resistance varies 'moderately', i.e in situations in which the local surface resistances stay below $r_{\mathrm{s}} \simeq 1000 \mathrm{~s} \mathrm{~m}^{-1}$ or in which less than half of the grid area is covered with very large $r_{s}$

\section{CONCLUSIONS}

Four models of effective drag coefficient for calculating the surface momentum flux over flat, but heterogeneous, terrain have been discussed. The models are compared by use of a micro-scale model which simulates the response of a surface-layer flow to varying boundary conditions in detail. It is found that sufficiently accurate estimates of surface momentum flux can be obtained by averaging the square of friction velocity or just the friction velocity itself based on a blending height. The concept of a blending height has been proposed earlier by Wieringa (1986) and Mason (1988). In this study it is suggested that the blending height should be defined as a height at which the sum of deviations of mean flow from local equilibrium and from horizontal homogeneity attains a minimum. This height is of the order of the diffusion height scale which forms a scale for the height up to which the mean flow can be influenced by vertical diffusion.

In analogy to the parametric drag coefficients parametric transfer coefficients of scalar admixtures are proposed. Again, averaging of the turbulent concentration scale or the product of turbulent concentration scale and friction velocity at the blending height gives the best estimates of the effective transfer coefficient The former average is more easily evaluated than the latter and, thus, preferred as parametric transfer coefficient.

The errors of the parametric drag coefficient and parametric transfer coefficient of scalar admixtures proposed in this study are within $\pm 10 \%$, which is quite small an error considering the imperfection in the determination of a roughness length itself. Therefore, it seems that the determination of surface fluxes is restricted by the uncertainty of local roughness length rather than by the parametric models.

The formulation of a parametric transfer coefficient of scalar admixtures has been extended to include surface conditions with varying surface resistance, which is a measure for the resistance against transport of an admixture into a surface, and with wind speed dependent laminar resistance, which basically gives the difference of roughness lengths of mean velocity and mean concentration of a scalar admixture. The effective transfer coefficient is difficult to parameterize for these surface conditions, because the local friction velocity should be known in order to obtain a correct estimate. Moreover, it is demonstrated that the dispersion of a scalar admixture is particularly sensitive to the variation of surface resistance, more than to perturbations of laminar resistance. For the case of wind speed dependent laminar resistance a practical 
parameterization of the effective transfer coefficient is found. For flow configurations with non-vanishing surface resistance, a parameterization is suggested which yields satisfactory results, except for terrain dominantly covered with patches of very strong surface resistances. This parameterization is a tentative proposal, because it is formulated for situations in which the surface resistance itself does not depend on the flow. On the other hand, it is well-known that the surface resistance (or stomatal resistance for vegetation) may strongly vary with environmental variables such as water vapour deficit and solar radiation (e.g. Stewart and DeBruin, 1985). Therefore, the investigation concerning changing surface resistance has to be regarded as a sensitivity study in order to isolate important mechanisms which further research has to focus on.

In earlier studies on parameterization of turbulent momentum transfer at the earth's surface, the influence of subgrid-scale motion other than micro-turbulence on the surface-layer flow has been neglected. Here, it is shown that the grid-averaged transfer coefficient (of momentum and of scalar admixtures) generally overestimates the effective transfer coefficient due to subgrid-scale correlation terms. Therefore, not the grid-average of the local ratio of surface flux and flow quantity, but the ratio of grid-averaged flux and grid-averaged flow quantity must be considered. The investigation of surface-layer transport by motions, which are larger than turbulent scale, but smaller than grid-scale, is not completed here. In a subsequent paper it will be studied how the grid-averaged momentum balance is affected by subgrid-scale motion which originates from heterogeneous terrain.

Acknowledgements-The author wishes to thank Prof. $\mathrm{H}$. Grassl, Max-Planck Institut für Meteorologie, Hamburg, and Dr D. Eppel, Forschungszentrum Geesthacht, for constructive critique and discussion.

\section{REFERENCES}

Anderson R. J. (1987) Wind stress measurements over rough ice during the 1984 Marginal Ice Zone Experiment. $J$. geophys. Res. 92, 6933-6941.

Anderson D. A. Tannehill J. C. and Pletcher R. H. (1984) Computational Fluid Mechanics and Heat Transfer. McGraw-Hill, New York.

Andre J.-C. and Blondin C. (1986) On the effective roughness length for use in numerical three-dimensional models. Boundary-Layer Met. 35, 231-245.
Beljaars A. C. M., Schotanus P. and Nieuwstadt F. T. M. (1983) Surface layer similarity under nonuniform fetch conditions. J. clim. appl. Met. 22, 1800-1810.

Brutsaert W. (1979) Heat and mass transfer to and from surfaces with dense vegetation or similar permeable roughness. Boundary-Layer Met. 16, 365-388.

Claussen M. (1987) The flow in a turbulent boundary layer upstream of a change in surfa 2 roughness. BoundaryLayer Met. 40, 31-86.

Claussen M. (1988) Models of eddy viscosity for numerical simulation of horizontally inhomogeneous surface layer flow. Boundary-Layer Met. 42, 337-369.

Claussen M. (1989) Neutral surface layer flow over isolated roughness strips. Boundary-Layer Met. 48, 431-442.

Garratt J. R. (1977) Aerodynamic roughness and mean monthly surface stress over Australia. CSIRO Aust. Div. Atmos. Phys. Tech. Pap. 29, 1-19.

Hasse L. (1976) A resistance-law hypothesis for the nonstationary advective planetary boundary layer. BoundaryLayer Met. 10, 393-407.

Hicks B. B. (1985) Application of forest-atmosphere turbulent exchange information. In The Forest-Atmosphere Interaction, pp. 631-644. D. Reidel, Dordrecht.

Hinze H. O. (1975) Turbulence. McGraw-Hill, New York. Jackson N. A. (1976) The propagation of modified flow downstream of a change in roughness. $Q$. Jl R. met. Soc. 102, 924-933.

Jackson P. S. and Hunt J. C. R. (1975) Turbulent flow over a low hill. Q. Jl R. met. Soc. 101, 929-955.

Louis J. F. (1979) A parametric model of vertical eddy fluxes in the atmosphere. Boundary-Layer Met. 102, 924-933.

Lumley J. L. and Panofsky H. A. (1964) The Structure of Atmospheric Turbulence. Interscience Publ., New York.

Mahrt L. (1987) Grid-averaged surface fluxes. Mon. Wea. Rev. 15, 1550-1560.

Malkus W. V. R. (1979) Turbulent velocity profiles from stability criteria. J. Fluid Mech. 90, 401-414.

Mason P. J.(1988) The formation of areally-averaged roughness lengths. Q. Jl R. met. Soc. 114, 399-420.

Petersen E. L. and Taylor P. A. (1973) Some comparisons between observed wind profiles at Risø and theoretical predictions for flow over inhomogeneous terrain. Q. $J l R$. met. Soc. 99, 329-336.

Peterson E. W. (1969) Modification of mean flow and turbulent energy by a change in surface roughness under condition of neutral stability. $Q$. $J l R$. met. Soc. 95, $561-575$.

Stewart J. B. and DeBruin H. A. R. (1985) Preliminary study of dependence of surface conductance of Thetford forest on environmental conditions. The Forest-Atmosphere Interaction, pp. 91-104. D. Reidel, Dordrecht.

Taylor P. A. (1987) Comments and further analysis on effective roughness lengths for use in numerical threedimensional models. Boundary-Layer Met. 39, 403-418.

Tennekes H. and Lumley J. L. (1972) A First Course in Turbulence. The MIT Press, Cambridge.

Wieringa J. (1986) Roughness-dependent geographical interpolation of a surface wind speed averages. Q.Jl R. met. Soc. $112,867-889$. 